\title{
EVALUACIÓN DEL DESEMPEÑO LABORAL EN EL ÁREA CULTURAL DEL MUNICIPIO DE OAXACA DE JUÁREZ
}

\author{
Guillermo Trejo Carbajal Mtro. ${ }^{1}$, Rosendo Martínez Jiménez ${ }^{2}$, Hernando Camacho Camacho ${ }^{3}$, Ana Luz Ramos \\ Soto $^{4}$
}

Resumen-El presente trabajo de investigación refiere la estructura Organizacional y analiza la propuesta del sistema de evaluación del desempeño Laboral en la Dirección de Cultura y Espectáculos del municipio de Oaxaca de Juárez durante el periodo anual de 2017. La aplicación adecuada del modelo estructural metodológico formula una propuesta real de evaluación en el desempeño laboral en un área cultural municipal requiere fijar los pasos fundamentales para construir el diseño apropiado que lleve a soluciones favorables que coadyuven a tener altos rendimientos en el campo del trabajo, para tal efecto se aplicó el método descriptivo, que permitió auxiliar en el manejo de la realidad y la investigación temática y empírica. El objetivo es proponer una herramienta actual para mejorar el desempeño laboral: coaching y su proceso. Como resultado se logró la participación de los empleados y generación del sentido de pertinencia, compromiso y responsabilidad en el área cultural municipal.

Palabras clave - Evaluación, desempeño laboral, municipal y cultural.

\section{Introducción}

En la Dirección de Cultura y Espectáculos del Municipio de Oaxaca de Juárez, Oaxaca., se verifico la existencia de un programa que evalué el desempeño laboral de los empleados que prestan sus servicios en ese órgano municipal, que sirva para impulsar acciones de capacitación y adiestramiento por parte de consultorías externas o de personal interno contratado de mayor experiencia y antigüedad, con el objetivo de alcanzar mejores niveles de entrenamiento y productividad laboral.

Es una prioridad que las instituciones tengan una herramienta apropiada en el control de la gestión de sus áreas altamente competitivas y brindar un servicio de calidad a los usuarios, para tal efecto deberá implementar diversos modelos para la evaluación del desempeño de los trabajadores, es por ello que se recomienda recopilar la información integral para lograr una correcta evaluación a través de indicadores que mejoren la toma de decisiones y permitan conocer de manera más exacta las competencias profesionales del capital humano.

El personal evaluado en la institución obtendrá beneficios al ponerse en práctica la evaluación al desempeño laboral, podrá identificar aciertos y fallas, fortalezas, debilidades, diagnosticar problemas y alternativas de solución para el mejoramiento laboral, como consecuencia contribuirá todo ello a incrementar la eficacia en el desempeño de sus funciones.

El método de investigación aplicado es la observación directa intersubjetiva, basado en el principio de observaciones repetidas, de las respuestas el observador debe producir los mismos datos, información que se traduce en ser más precisa, verídica y oportuna sobre el desempeño de los funcionarios y empleados municipales. De acuerdo con lo que expresa Tamayo y Tamayo, se hace referencia explícitamente a la percepción visual, y se emplea para indicar todas las formas de percepción utilizadas para el registro de respuestas tal como lo perciben nuestros sentidos (Tamayo y Tamayo, Mario, 2014)

También aplica la técnica de observación indirecta al corroborar los datos tomados de otros, como son los testimonios orales de los empleados y directivos de órgano público municipal.

Refiere antecedentes aplicados en otras Instituciones de la Administración Pública en los niveles Estatal y Federal sobre el cómo se practica la evaluación al desempeño laboral, con modelos diversos tales como programas

\footnotetext{
${ }^{1}$ Guillermo Trejo Carbajal es Profesor Investigador de tiempo completo de la Facultad de Contaduría y Administración (FCA) de la Universidad Autónoma "Benito Juárez" de Oaxaca (UABJO), integrante del cuerpo académico en consolidación "Emprendedores" UABJO-CA-46. guillermo.carbajal@ hotmail.com

${ }^{2}$ Rosendo Martìnez Jiménez es Profesor Investigador de tiempo completo de la Facultad de Contaduría y Administración (FCA) de la Universidad Autónoma "Benito Juárez" de Oaxaca (UABJO), integrante del cuerpo académico en consolidación "Emprendedores" UABJO-CA-46. martinezjmz 10@ hotmail.com

${ }^{3}$ Hernando Camacho Camacho es líder de investigación de la Maestría en Calidad y Gestión Integral del convenio USTA-ICONTEC, Colombia. hernandocamacho@usantotomas.edu.co

${ }^{4}$ Ana Luz Ramos Soto Profesora Investigadora de tiempo completo de la Facultad de Contaduría y Administración (FCA) de la Universidad Autónoma "Benito Juárez" de Oaxaca (UABJO), integrante del cuerpo académico en consolidación

"Emprendedores”UABJO-CA-46. analuz_606@yahoo.com.mx
} 
de calidad, eficiencia y valoración de méritos para motivar y sensibilizar a que los servidores públicos procuren un desarrollo puntual en las competencias laborales asignadas en el puesto que tienen bajo su responsabilidad.

Incorpora al Coaching como una herramienta recomendable actual en las organizaciones, describe las formas de entrenamiento que se podrán aplicar para que con un notable esfuerzo superen su actividad laboral.

Incorpora diversos métodos de evaluación al desempeño laboral, entre ellos los de escala gráfica, elección forzada y de investigación de campo.

\section{Descripción del Método}

El método de investigación aplicado es la observación directa intersubjetiva, basado en el principio de observaciones repetidas, de las respuestas el observador debe producir los mismos datos, información que se traduce en ser más precisa, verídica y oportuna sobre el desempeño de los funcionarios y empleados municipales. De acuerdo con lo que expresa Tamayo y Tamayo, se hace referencia explícitamente a la percepción visual, y se emplea para indicar todas las formas de percepción utilizadas para el registro de respuestas tal como lo perciben nuestros sentidos (Tamayo y Tamayo, Mario, 2014). También aplica la técnica de observación indirecta al corroborar los datos tomados de otros sujetos, como son los testimonios orales de los empleados y directivos de órgano público municipal.

Refiere antecedentes aplicados en otras Instituciones de la Administración Pública en los niveles Estatal y Federal sobre el cómo se practica la evaluación al desempeño laboral, con modelos diversos tales como programas de calidad, eficiencia y valoración de méritos para motivar y sensibilizar a que los servidores públicos procuren un desarrollo puntual en las competencias laborales asignadas en el puesto que tienen bajo su responsabilidad.

Incorpora al Coaching como una herramienta recomendable actual en las organizaciones, describe las formas de entrenamiento que se podrán aplicar para que con un notable esfuerzo superen su actividad laboral.

Se enfoca a los indicadores de desempeño que se utilizan en el sector público para tener un análisis más integral y detallado sobre la aplicación en la evaluación del desempeño laboral de los servidores públicos que se encuentran adscritos en la Dirección de Cultura y Espectáculos.

Incorpora diversos métodos de evaluación al desempeño laboral, entre ellos los de escala gráfica, elección forzada y de investigación de campo. Se demuestra la relevancia del papel que tiene la evaluación del desempeño laboral y que por medio de la práctica del coaching en las instituciones públicas presentan una mejora notable en la prestación de los servicios de los empleados de la administración pública municipal.

\section{Descripción de la evaluación del desempeño laboral.}

\section{Marco Teórico}

El desempeño de cada persona requiere de una evaluación. Esta actividad no solo permite estimar la forma en que cada persona cumple con sus responsabilidades, sino que indica también las actividades de los recursos humanos para que las realicen de manera adecuada. Un desempeño deficiente puede indicar que la selección, la capacitación o las actividades de desarrollo deben revisarse o también pueden existir problemas en relación del personal con la organización.

Como resultado de dicha evaluación los empleados deben recibir una compensación que puede ser en forma de sueldos y salarios. Cabe mencionar que una función de la administración de recursos humanos es la evaluación al desempeño teniendo así antes; la incorporación de personal, administración de sueldos, prestaciones y servicios, educación y capacitación, comunicación, liderazgo, motivación, control y evaluación del desempeño. Mientras tanto el proceso del área de recursos humanos es; reclutamiento y selección, inducción, remuneraciones, capacitación, motivación y clima laboral. "El sistema de evaluación al desempeño es un proceso riguroso y científico que permite obtener información de las aportaciones de cada uno de los empleados en un periodo determinado de tiempo, así como del grado de cumplimiento de los objetivos asignados. Este periodo sistemático de evaluación determina la eficiencia con que cada una de las personas realiza sus cometidos y funciones y se basa en un modelo participativo en la definición del propio sistema." (http://www.acotex.org/wpcontent/uploads/2013/07/web_20121010_Retribucion_2011_Recursos_Humanos.pdf, s.f.)

Los sistemas de evaluación deben estar directamente relacionados con el puesto y ser prácticos y confiables. Se refiere a que el sistema solo califica elementos de importancia para lograr la labor con éxito del individuo, por el contrario, si la evaluación no se relaciona con el puesto carece de validez, y se entiende que la evaluación es práctica, cuando el evaluador y el empleado la comprenden con facilidad. Además, un buen sistema de evaluación puede también identificar problemas en el sistema de información sobre recursos humanos. Las personas que se desempeñan de manera ineficiente pueden poner en evidencia procesos equivocados o pobres de selección, 
orientación y capacitación. De modo importante, el desempeño inferior al esperado puede identificar que el diseño del puesto o los desafíos externos no han sido considerados en todas sus facetas.

Por lo tanto, para poder llevar a cabo la evaluación del desempeño, se deben tener como base los manuales y lineamientos establecidos en la institución, conocer su filosofía, estructura y objetivos lo cual la hace acreedora a que se efectué solo en esa institución.

Como nos menciona el autor Davis (2000) "Una organización no puede sencillamente adoptar cualquier sistema de evaluación al desempeño. El sistema debe ser válido, confiable y aceptado..." “... Si las normas para la evaluación al desempeño no se basan en los elementos relacionados con el puesto, pueden traducirse a resultados imprecisos o subjetivos". En conclusión, se puede decir que la evaluación es el proceso mediante el cual se estima, aprecia y valora el rendimiento del individuo en una determinada área o departamento para después ser calificado y medido a través de la eficiencia. Mediante el cual es posible localizar problemas de supervisión de personal y de la integración del colaborador a la organización.

Beneficios de la evaluación del desempeño, cuando un programa de evaluación del desempeño está bien planeado, coordinado y desarrollado, proporciona beneficios a corto, mediano y largo plazo, en general, los principales beneficiarios son el individuo, el gerente, la organización y la comunidad. Para el primero, conoce las reglas de juego, es decir, los aspectos, de comportamiento y de desempeño que más valora la empresa en sus empleados, cuáles son las expectativas de su jefe acerca de su desempeño, sus fortalezas y debilidades, según la evaluación del jefe (programas de entrenamiento, capacitación, etc.), y las que el propio subordinado deberá tomar por su cuenta (autocorrección, mayor esmero, mayor atención al trabajo, cursos por su propia cuenta, etc.). Para el segundo, la gerencia permite evaluar mejor el desempeño y el comportamiento de los subordinados, con base en las variables y los factores de evaluación y, sobre todo, contar con un sistema de medición capaz de neutralizar la subjetividad, propone medidas y disposiciones orientadas a mejorar el estándar de desempeño de sus subordinados. En el tercero, la organización podrá evaluar su potencial humano a corto, mediano y largo plazo, definir la contribución de cada empleado. Puede identificar a los empleados que necesitan actualización o perfeccionamiento en determinadas áreas de actividad, y seleccionar a los empleados que tienen condiciones para ascender o transferirlos. Puede dar mayor dinámica a su política de recursos humanos ofreciendo oportunidades a los empleados, estimulando la productividad y mejorando las relaciones humanas del trabajo.

El Coaching como herramienta actual en las organizaciones, el termino Coaching es un anglicismo que procede del término inglés to coach (entrenar), proviene del inglés y del francés en el ámbito empresarial el coaching se ha convertido en una herramienta útil para manejar todos los cambios que se están generando en el mercado y en el escenario global. El coaching según Whitmore (2003) consiste en "liberar el potencial de una persona para incrementar al máximo su desempeño. Así, etimológicamente, el término coach deriva de un medio de transporte, y de alguna manera el coaching sirve para transportar a las personas del lugar donde están hasta el lugar donde desean estar, el proceso de coaching es relativamente novedoso en el campo laboral; sin embargo, sus orígenes son ancestrales y puede decirse que se practica desde la época de los filósofos griegos; la técnica usada por Sócrates con sus discípulos puede considerarse uno de los antecedentes principales del coaching.

En general, la disciplina denominada actualmente coaching ayuda a realizar cambios personales y profesionales, y simultáneamente desarrolla una gran variedad de posibilidades de actuación; se basa en que el sujeto aprenda a desafiar sus creencias limitantes, así como los obstáculos o interpretaciones obsoletas que le impiden alcanzar el éxito, es un juego empresarial que concentra escenarios y actores que compiten para ganar. La idea es que durante este juego se mezclen experiencias y enseñanzas que permitan a cada jugador llegar a un triunfo. Todo esto será dirigido por un coach que será un aporte único de liderazgo personal; este coach se encargará de guiar el talento humano de la empresa para llegar a realizar y convertir cada meta en un triunfo.

Análisis y propuesta de un sistema de evaluación del desempeño laboral en la Dirección de Cultura y Espectáculos

Con el objetivo de aplicar el tema expuesto en este trabajo de investigación a un caso real, se ha elegido una organización en la que, a partir de sus características, se diseña un proceso de evaluación de desempeño basado en el método de investigación de campo. Se cuenta con el actual organigrama en el departamento: 
Figura 1. Estructura Organizacional de la Dirección de Cultura y Espectáculos.

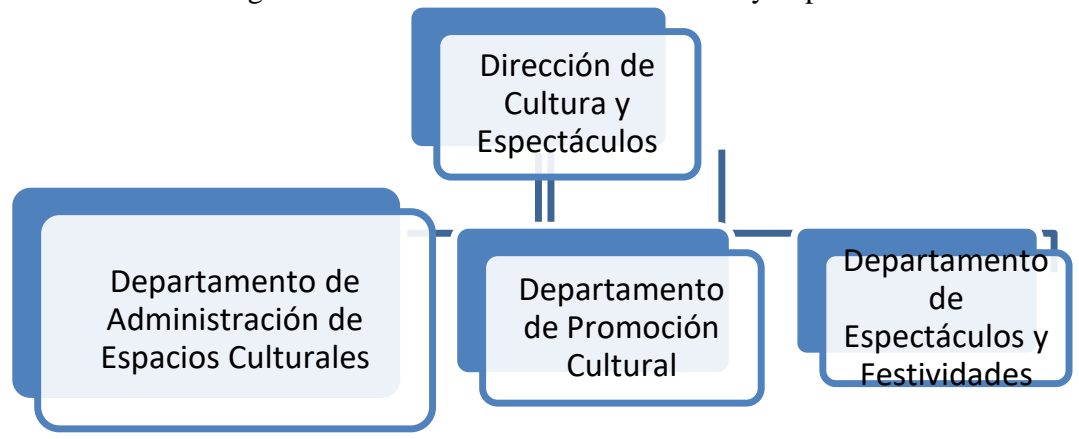

Fuente: Manual de Organización de la Secretaria de Desarrollo Humano

La evaluación que se aplica actualmente en la institución se basa en opiniones del jefe directo, ya que no se cuenta con una evaluación estructurada y amparada con entrevistas, exámenes o documentos que nos permitan conocer las capacidades reales del personal. A fin de año los empleados obtienen un bono económico, como premio por su desempeño, sin embargo, no se basa en una evaluación formal del desempeño, sino sólo en el criterio del Director General.

Análisis y desarrollo de una propuesta de evaluación de desempeño, el objetivo de esta sección del trabajo de investigación es la de diseñar un procedimiento de evaluación de desempeño por el método de investigación de campo y conjugándolo con el Coaching para obtener beneficios más satisfactorios para la institución a la par de los colaboradores.

Escalas de medición, Para calcular el desempeño de cada empleado desde el punto de vista de objetivos y competencias, debemos definir las escalas de medición que se aplicarán. Teniendo en cuenta los objetivos que supervisor-empleado definen a principio de año, y que guían el desempeño del empleado para todo ese periodo, se define la siguiente escala para su medición:

1- Supera ampliamente el cumplimiento del objetivo definido (100\%).

2- Supera el cumplimiento del objetivo definido (75\%).

3- Alcanzó el objetivo (50\%)

4- Estuvo cerca de alcanzar el objetivo (25\%)

5- No alcanzó el objetivo (0\%).

Obtener una calificación de "1" en la evaluación de los objetivos implica no sólo haberlo cumplido de la manera que fue prevista, sino superando ampliamente el resultado esperado. En cambio, una calificación de " 4 " corresponde a un objetivo que estuvo cerca de ser alcanzado o que fue alcanzado, pero fuera de tiempo o superando la restricción presupuestaria. Por último, la calificación " 3 " implica haber cumplido el objetivo en el tiempo y forma establecidos.

Actualmente existen 16 trabajadores en la Dirección de Cultura y Espectáculos, de los cuales 1 es de confianza y 15 son de base, por cuestiones políticas, este método se aplica al personal de base, para definir el desempeño de manera individual, basados en la escala de medición, se crea un formato de evaluación al desempeño, que se aplicara a cada jefe de departamento y en colaboración con ellos, se revisaran los resultados obtenidos de cada una de las personas a su cargo, para tal efecto se aplico un formato de evaluación del desempeño laboral del personal de base y confianza adscrito en la Dirección de Cultura y Espectáculos de la Secretaria de Desarrollo Humano del Municipio de Oaxaca de Juárez.

Figura 2. Resultados del Desempeño del personal de la Dirección de Cultura y Espectáculos.

\begin{tabular}{|c|c|r|r|r|r|r|}
\hline \multicolumn{2}{|c|}{ Dirección de Cultura y Espectáculos. } & \multicolumn{5}{|c|}{ ESCALA DE MEDICION \% } \\
\hline \multirow{2}{*}{ DEPARTAMENTO } & CATEGORÍA & $\begin{array}{r}\mathbf{1 0 0} \\
\mathbf{\%}\end{array}$ & $\begin{array}{r}\mathbf{7 5} \\
\mathbf{\%}\end{array}$ & $\begin{array}{r}\mathbf{5 0} \\
\mathbf{\%}\end{array}$ & $\begin{array}{r}\mathbf{2 5} \\
\mathbf{\%}\end{array}$ & $\begin{array}{r}\mathbf{0} \\
\mathbf{\%}\end{array}$ \\
\hline \multirow{3}{*}{$\begin{array}{c}\text { Departamento de Administración } \\
\text { de Espacios Culturales. }\end{array}$} & JEFE & & $*$ & & & \\
\cline { 2 - 7 } & Administrador & & $*$ & & & \\
\cline { 2 - 7 } & Administrador & & & $*$ & & \\
\cline { 2 - 7 } & Administrador & & $*$ & & & \\
\cline { 2 - 7 } & Administrador & & & & $*$ & \\
\hline Departamento de Promoción & JEFE & & & $*$ & & \\
\hline
\end{tabular}




\begin{tabular}{|c|c|c|c|c|c|c|}
\multirow{2}{*}{ Cultural. } & Promotor & $*$ & & & & \\
\cline { 2 - 6 } & Promotor & & & $*$ & & \\
\hline & Promotor & & & & $*$ & \\
\hline & Promotor & & $*$ & & & \\
\hline & JEFE & & $*$ & & & \\
\hline \multirow{5}{*}{$\begin{array}{c}\text { Departamentos de Espectáculos y } \\
\text { Festividades. }\end{array}$} & Coordinador & & & & $*$ & \\
\hline & Coordinador & & $*$ & & & \\
\hline & Coordinador & & & $*$ & & \\
\hline & Coordinador & $*$ & & & & \\
\hline
\end{tabular}

Fuente: Elaboración propia.

En la tabla de la figura 2 se reflejan los porcentajes de acuerdo a la tabla de escalas de medición del desempeño que en conjunto con los jefes directos de cada área se asignaron a cada colaborador, notando que ninguna persona ocupo el $0 \%$, es decir que ninguna persona incumplió con los objetivos que les establece la institución, en base a las actividades que desempeña cada colaborador a su cargo, uso de papelería y materiales de trabajo, de los cuales cada jefe tiene control sobre ellos, al evaluar el desempeño individual, también se basaron en asistencias y la disposición del cumplimiento en las tareas encomendadas.

Analizando los resultados obtenidos de la información anterior, se observa que el 17\% supera ampliamente el cumplimiento del objetivo definido, $33 \%$ supera el cumplimiento del objetivo definido, $25 \%$ alcanzó el objetivo, $25 \%$ estuvo cerca de alcanzar el objetivo $0 \%$ no alcanzó el objetivo.

Figura 3. Grafica de resultados del Desempeño del personal de la Dirección de Cultura y Espectáculos.

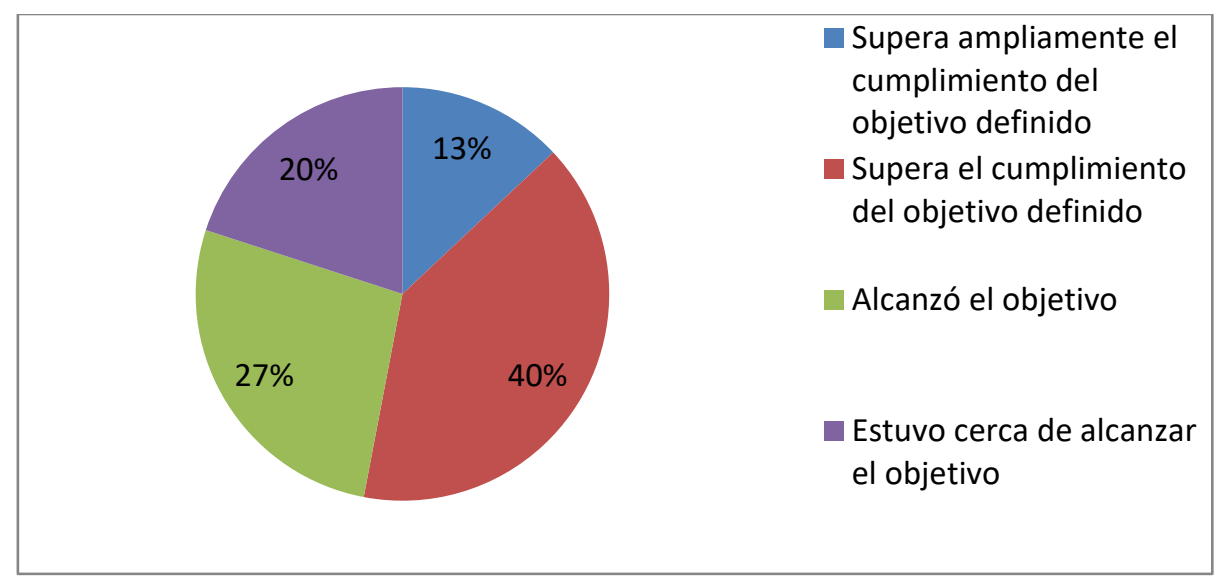

Fuente: Elaboración propia.

Esto permite determinar que personas se asignarán como coach y quienes serán coacheados para con ello elevar la calidad de los trabajadores y de la institución.

Como se comentó anteriormente, en diciembre cada trabajador percibe un bono que equivale a un mes neto de pago por su productividad obtenida durante el año, en este punto ya no será la misma compensación, la propuesta es determinar un porcentaje mayor para quienes son coach y un porcentaje menor para quienes son coacheados. Es importante mencionar que el coaching es una vía rápida para comenzar a aprender de otra manera, y es útil para realizar cambios tanto en el ámbito profesional como personal. Conduce a eliminar los obstáculos que impiden alcanzar el máximo potencial de una persona, es una forma para adentrarse en otra "forma de mirar", y con perseverancia desde allí ir dando más significado, coherencia y bienestar a la vida. Desde esta óptica, el coaching aplicado en las organizaciones constituye una herramienta poderosa para crear una cultura de alto desempeño y rica en retroalimentación que se aplica como último paso en el proceso de evaluación, es muy útil en las organizaciones actuales y se considera como tecnología de desempeño humano en las dependencias públicas poco se practica como tal este sistema y es un complemento importante en el proceso de aprendizaje laboral, aplicarlo a una institución sería un logro para sus controles. 


\section{Conclusiones}

En el trayecto de esta investigación concluimos que las instituciones del sector público tienden a enfocar la evaluación del desempeño al área financiera y al presupuesto que se le asigna a cada sector dejando fuera la importancia de la competitividad y productividad de cada colaborador, cuentan con sistemas poco supervisados de evaluación al desempeño personal, por tal motivo hacer un análisis cualitativo, permite tener un panorama más amplio sobre las opiniones de cada colaborador y así tomar decisiones que permitan mejorar el ambiente laboral.

Se debe considerar que la evaluación del desempeño no solo es para el nivel operativo, es también aplicado a nivel ejecutivo y directivo, existen diferentes tipos de evaluación dentro de las instituciones públicas, pero la interna es la que permite conocer detalladamente la situación en la que se encuentra la institución en cuanto a competitividad y calidad.

Durante la aplicación de la evaluación del desempeño se obtiene información que permite tomar decisiones y promover un sistema mejorado, en una institución pública favorece el control de la misma y obtiene beneficios tanto para la institución como para el nivel directivo, ejecutivo y operativo.

Con base en los parámetros de desempeño, se permite establecer los estándares que nos lleven a tomar mejores decisiones actualmente, el coaching es una herramienta que facilita el proceso de aprendizaje y aplicado de forma correcta permite compensar de manera justa al personal por eso se recomienda implementar el modelo de evaluación aplicado en la Dirección de Cultura y Espectáculos.

La investigación realizada genero esta propuesta de manera conjunta con algunos colaboradores de la institución, pero las políticas internas que rigen a esta organización pública municipal solo aprobaron el proceso de la revisión, quedando pendiente su implantación.

\section{BIBLIOGRAFIA}

BALDERAS, M. (1995). Administración de Recursos Humanos. Mexico: McGRAW-HILL. Chiavenato, i. (1988). administracion de recursos humanos. mexico: mc graw hill.

Davis, k. (2000). Administracion de personal y recursos humanos. mexico: McGrawll-Hill.

Davis, w. b. (2000). administracion de personal y recursos humanos. mexico: mc graw-hill.

España, J. L. (2010). Coachingactual.com Socrates y TU presente. Lito-Grapo S.A de S.V.

Espinosa, l. f. (1999). administracion de recursos humanos para el alto desempeño. mexico: trillas.

F.J, C. (2004). Coaching, mitos y realidades . Madrid: Pearson Educacion.

Gomez, G. R., Gil Flores, J., \& Garcia Jimenez, E. (1996). Metodologia de la Investigacion Cualitativa. España:

Ediciones Aljibe .

Larriera, E. (2005). Coaching mayéutico o cómo ser mastro: la herencia de Sócrates en las organizaciones.

Loflan, L. y. (1995).

M. Williams, C. M. (2002). Estudio sobre los 8 comportamientos clave del coach.

OCDE. (1998). La promoción del uso del Programa Evaluación rendición de cuentas en las organizaciones públicas.

Olea, R. V. (2011). El coaching organizacional y sus aplicaciones. Mexico: Pearson.

Sampieri, R. H., Fernandez Collado, C., \& Baptista Lucio, P. (2006). Metodologia de la Investigación. Mexico: McGraw-Hill.

Shaun Tyson, A. Y. (1989). Administracion de personal. Mexico: Trillas.

sherma, b. g. (2001). administracion de recursos humanos. mexico: international thomson editores.

Valencia, J. R. (2007). Administracion Moderna de Personal (7ma ed.). Mexico: CENGAGE.

Villegas, J. M. (1988). Administración de Personal. Mexico: texto s.r.l.

W. Goad, T. (1992). El profesional en el desarrollo de recursos humanos. mexico: continntal s.a. de c.v.

Whitmore, J. (2003). Coaching. España: McGraw-Hill.

William B. Werther, J. K. (1987). Administracion de personal y recursos humanos. Mexico: Mc Graw Hill.

http://www.acotex.org/wp-content/uploads/2013/07/web_20121010_Retribucion_2011_Recursos_Humanos.pdf. (s.f.).

http://www.ehu.eus/documents/1393006/1446455/Gestion_de_los_rrhh_en_empresas_innovadoras.pdf. (s.f.). http://www.fvet.uba.ar/postgrado/especialidad/power_taller.pdf. (s.f.).

http://www.sociedadelainformacion.com/21/recursos.pdf. (s.f.) 